\title{
Appearance of an inverted appendix on virtual colonoscopy
}

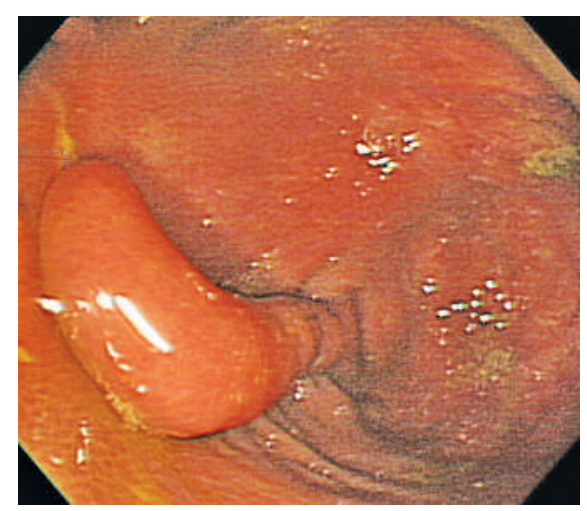

Figure 1 A 55-year-old woman with systemic lupus erythematosus, who had been followed up regularly for 7 years, visited our hospital complaining of chronic abdominal fullness, constipation, and stringy stools. She had a history of a total hysterectomy for uterine prolapse at the age of 38. At colonoscopy we found a 3-cm-long tubular structure, with normal-appearing mucosa, in the cecum; histopathological examination showed colonic mucosa with mild inflammation. A diagnosis of inverted appendix was made.

\section{A.-L. Chou ${ }^{1}$, C.-W. Lin' ${ }^{2}$ K.-C. Tseng1}

${ }^{1}$ Department of Internal Medicine, Buddhist Dalin Tzu Chi General Hospital, Chiayi, Taiwan

${ }^{2}$ Department of Radiology, Buddhist Dalin Tzu Chi General Hospital, Chiayi, Taiwan

Endoscopy_UCTN_Code_CCL_1AD_2AC Endoscopy_UCTN_Code_CCL_1AD_2AJ

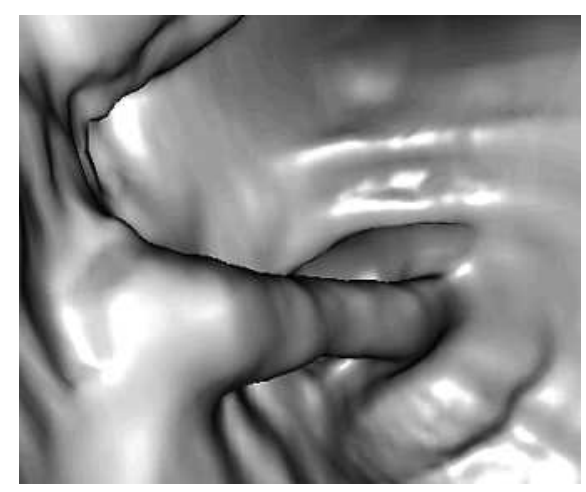

Figure 2 Virtual colonoscopy performed immediately after colonoscopy revealed a threedimensional tubular structure, similar to that seen on colonoscopy. Inverted appendix should be considered in the differential diagnosis of cecal polyps.

Corresponding Author

\section{K.-C. Tseng, M.D.}

Department of Internal Medicine Buddhist Dalin Tzu Chi General Hospital 2 Min Sheng Road

Dalin

Chiayi

Taiwan

Fax: $\quad+886-5-2648006$

E-mail: dennis0.tseng@msa.hinet.net 